\title{
Síndrome de Tako-Tsubo en perioperatorio: Una variante rara. Reporte de caso
}

\author{
Tako-Tsubo syndrome in perioperative: A rare variant. \\ Case report \\ Jesús H. Tovar1,3, Jorman H. Tejada²,3, César D. Galindo4, Carlos E. Melo4, Hansel S. Lis
}

\begin{abstract}
Tako-Tsubo syndrome or stress-induced cardiomyopathy has been described as a transient left ventricular apical dyskinesia with chest pain and STsegment elevation on electrocardiogram in the absence of obstructive lesions in coronary arteriography, most commonly in perimenopausal women. It is a rare pathology, with five reported variations and debated pathophysiological mechanisms. The current most accepted theory is a surge of catecholaminergic discharge, associated with genetic predisposition and an emotional or physical stress factor as trigger. We report a case of a 27 -year-old woman with a history of uncontrolled asthma and a brother with early heart disease. She underwent appendectomy under general anesthesia and immediately after extubation, she presented a drop in oxygen saturation, respiratory distress, oppressive chest pain, severe arterial hypotension and acute pulmonary edema, requiring reintubation. ST depression on electrocardiogram, positive troponins, coronary arteriography without obstructive lesions, and mid-ventricular hypokinesia was observed in the complementary cardiac studies. Based in these findings, stress cardiomyopathy was diagnosed. The patient progressed favorably, four months after discharge, the patient remained asymptomatic with no changes in the cardiac studies.
\end{abstract}

\section{Key words:}

Tako-Tsubo, perioperative transient dyskinesia

1 MD. Anestesiólogo. Hospital Universitario Hernando Moncaleano Perdomo. Neiva, Colombia.

2 MD. Neuroanestesiólogo. Coordinador Servicio de Anestesiología. Hospital Universitario Hernando Moncaleano Perdomo. Neiva, Colombia.

3 Docente Asistente Posgrado de Anestesiología y Reanimación. Universidad Surcolombiana. Neiva, Colombia.

4 Médico. Residente de Anestesiología y Reanimación. Universidad Surcolombiana. Neiva, Colombia.

Fecha de ingreso: 10 de agosto de 2020

Fecha de aceptación: 12 de octubre de 2020

\section{ORCID}

0000-0003-1269-4225

\section{Correspondencia:}

Jesús H. Tovar

jeshertoc@hotmail.com 


\section{RESUMEN}

El síndrome de Tako-Tsubo o miocardiopatía por estrés es descrito como una discinesia apical transitoria con cuadro de dolor torácico típico y electrocardiograma con elevación del segmento ST ante ausencia de lesiones en arteriografía coronaria, presentada principalmente en mujeres perimenopáusicas. Se reconoce como una patología poco frecuente, con 5 variaciones reportadas y de la que aún se debaten los mecanismos fisiopatológicos, siendo la descarga catecolaminérgica, asociada a predisposición genética y un factor emocional y/o físico desencadenante, la teoría más aceptada hoy en día. Se presenta el caso de una mujer de 27 años con antecedente de asma no controlada y hermano con enfermedad cardiaca temprana, que es llevada a apendicectomía abierta bajo anestesia general e inmediatamente a la extubación presenta caída de la saturación de oxígeno, dificultad respiratoria, refiere dolor torácico opresivo, hipotensión arterial severa y edema agudo de pulmón, con requerimiento de nueva intubación; electrocardiograma con infradesnivel del ST, troponinas positivas, arteriografía coronaria sin lesiones e hipocinesia medioventricular, con lo que se diagnostica miocardiopatía por estrés. Evolución favorable y egreso, y seguimiento a los 4 meses con exámenes normales.

\section{Palabras clave:}

Tako-Tsubo, disquinesia transitoria perioperatoria

\section{Introducción}

I síndrome de Tako-Tsubo, también denominado miocardiopatía por estrés o síndrome de corazón roto, fue reportado por primera vez en Estados Unidos en 1986 en una paciente femenina, a quien se le reportó anormalidades de la pared ventricular medial y apical con cambios electrocardiográficos y elevación de marcadores de injuria cardiaca, sin evidencia de lesión arterial, que resolvió en semanas[1]. Más adelante, Sato et al. en 1990, reporta un caso de discinesia apical transitoria asociado a estrés emocional, el cual denominó "Tako-Tsubo", por la imagen ecocardiográfica similar a la maceta de cuello estrecho y base ancha usada como trampa para atrapar pulpos por los pescadores en Japón. El cuadro clínico no discrepa con los síntomas producidos por un síndrome coronario; sin embargo, no se presentan lesiones en la coronariografía, aunque sí se observa hipocinesia apical en forma de balón del ventrículo izquierdo[2].

Se presenta un caso de una mujer joven en posoperatorio inmediato de apendicetomía abierta, quien presenta caída severa de la saturación de oxígeno, edema pulmonar agudo, con dolor torácico típico, diagnosticada con síndrome Tako-Tsubo en variante de discinesia medioventricular, por cardiología invasiva.

\section{Presentación del caso}

Al hospital ingresa mujer de 27 años de edad con antecedente de asma no controlada y hermano con enfermedad coronaria a los 34 años. Llega por cuadro clínico de dolor abdominal de 12 h compatible con apendicitis aguda, por lo que se lleva a apendicetomía abierta. Valoración preanestésica: estado ASA $2 \mathrm{U}$ sin otros hallazgos. Se inicia anestesia general con secuencia de intubación rápida con inducción con lidocaína 40 mg, propofol 150 mg, fentanil 170 mcg, ketamina $10 \mathrm{mg}$ y rocuronio $25 \mathrm{mg}$. Intubación orotraqueal en primer intento sin complicaciones. Monitoria básica dentro de parámetros normales. Mantenimiento anestésico con remifentanil a 0,2 mcg/kg/min, y sevoflurano con MAC de 1.2.

Procedimiento quirúrgico de 32 minutos sin complicaciones. Se realiza reversión de relajación neuromuscular con sugammadex 200 mg, y se procede a despertar, a la paciente con parámetros ventilatorios adecuados. Posterior a la extubación, con la paciente despierta se presenta hipertensión arterial y refiere dolor torácico típico, seguido a desaturación severa $\left(\mathrm{SOP}_{2}<80 \%\right)$ con dificultad respiratoria que no resuelve con oxígeno por máscara facial y caída severa de la presión arterial (PAM < $40 \mathrm{mmHg}$ ), se auscultan estertores generalizados en ambos campos pulmonares; el anestesiólogo procede a nueva intubación, pre- 
via inducción e inicio de infusión de soporte vasopresor con norepinefrina $(0,5 \mathrm{mcg} / \mathrm{kg} / \mathrm{min})$. Paso de tubo endotraqueal al primer intento, con salida de líquido espumoso color salmón al paso del tubo.

El anestesiólogo diagnostica edema agudo de pulmón de origen cardiogénico por evento coronario posanestésico versus tromboembolismo pulmonar. Se estabiliza la paciente, y se traslada a unidad de cuidados intensivos (UCI) $1 \mathrm{~h}$ después a la nueva intubación, con soporte ventilatorio y hemodinámico doble (norepinefrina y dobutamina). Se toma un electrocardiograma de ingreso a UCI con taquicardia sinusal e infradesnivel del segmento ST en las derivaciones precordiales (Figura 1) y laboratorios: leucocitosis con neutrofilia, hemoglobina y plaquetas normales, función renal y tiroidea conservada, y troponinas mayores a 1.001 ng/ml. Gases arteriales: acidosis mixta no compensada con trastorno severo de la oxigenación e hipokalemia leve $\left(\mathrm{pH} 7.189, \mathrm{PO}_{2} 76, \mathrm{PaFi} 76, \mathrm{PCO}_{2}\right.$ $55,4, \mathrm{HCO} 320,7$, lactato 2,25 , sodio 143 , potasio

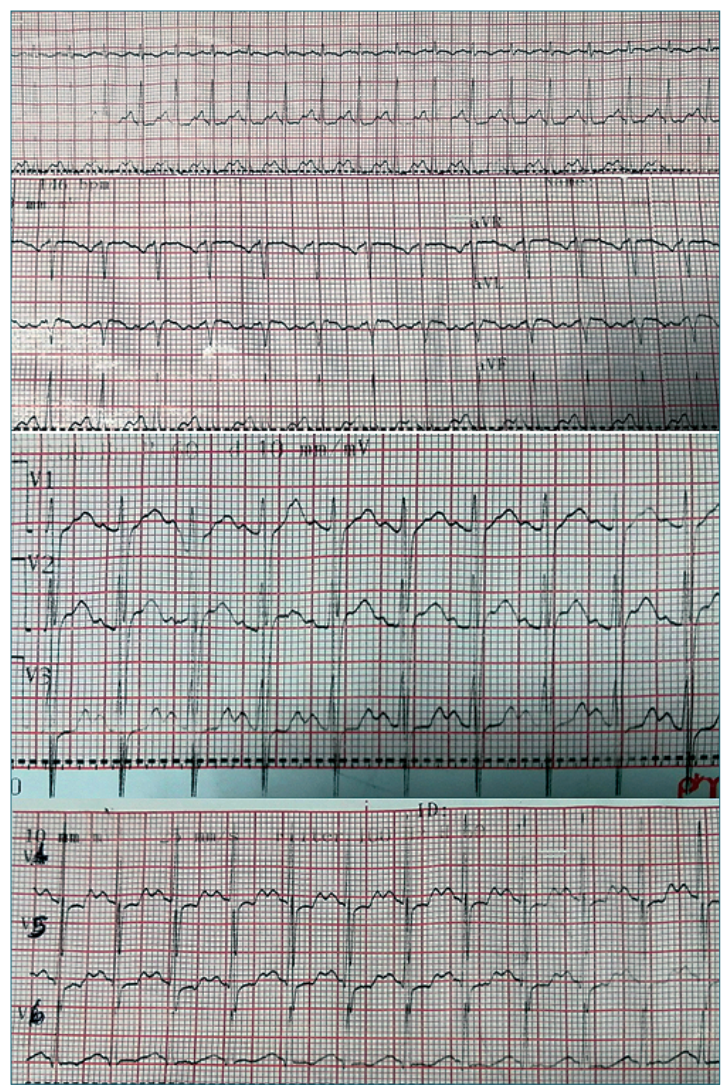

Figura 1. Electrocardiograma de ingreso a unidad de cuidados intensivos. Se observa taquicardia sinusal e infradesnivel del segmento ST en derivaciones precordiales V4-V6.

\section{3,06, calcio 1,15, cloro 103).}

Evolución clínica favorable con retiro de vasopresor a las 24 h y extubación sin complicaciones a las 48 h del ingreso a UCl con realización el mismo día de arteriografía coronaria por cardiología invasiva sin evidencia de lesión coronaria ni valvular, ventrículo izquierdo levemente dilatado e hipocinesia medioventricular, por lo cual se diagnostica síndrome de Tako-Tsubo (Figuras 2 y 3). Tres días posteriores a la cirugía se toma ecocardiograma transtorácico que reporta corazón estructuralmente normal con función sistólica y diastólica conservada, y fracción de eyección ventricular izquierda del 40\%.

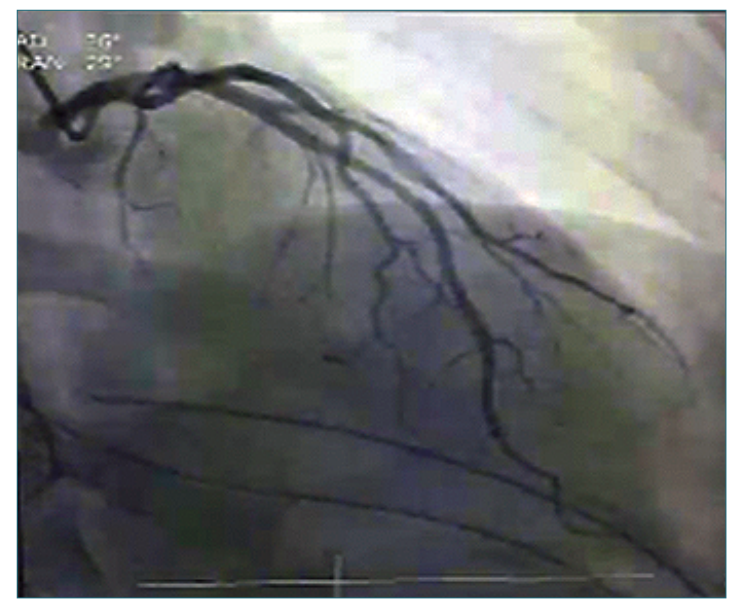

Figura 2. Coronariografía izquierda sin lesiones significativas.

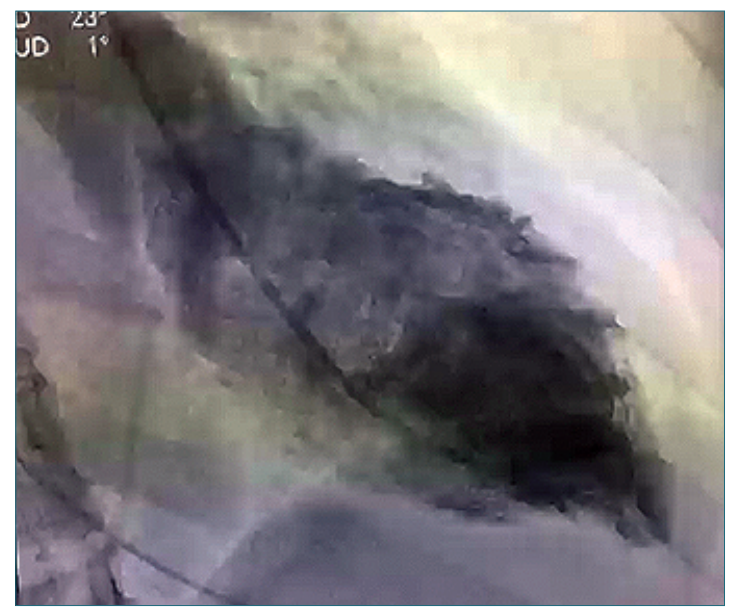

Figura 3. Ventriculografía durante la contracción ventricular. Imagen de Takot-Tsubo. 
La paciente presentó evolución clínica favorable, por lo que a los 5 días posteriores a la cirugía se dio orden de egreso hospitalario con control ambulatorio por cardiología con manejo farmacológico (carvedilol y enalapril). En el seguimiento a los 4 meses: Electrocardiografía normal y ecocardiograma transtorácico sin alteraciones con fracción de eyección normal, y troponinas negativas.

\section{Discusión}

El síndrome de Tako-Tsubo, miocardiopatía por estrés o síndrome de corazón roto, como se ha denominado, se define como un tipo especial de discinesia transitoria de las paredes del ventrículo izquierdo especialmente en la región apical, desencadenada por estrés emocional o físico. Fue reportada por primera vez en 1986 en Massachusets en una paciente con intento de suicidio $y$, posteriormente, aparente evento isquémico miocárdico sin lesión de arterias coronarias[1]; más adelante el Dr. Hikaru Sato en el Hospital de Hiroshima-Japón realiza el reporte de una paciente con anomalías del movimiento del ápex sin evidencia de lesión de arterias coronarias, y una "imagen de trampa de pulpo o Tako-Tsubo", por lo cual da el nombre, atribuyéndolo en ese momento a un espasmo coronario multivaso[2].

Esta singular entidad caracterizada por imitación clínica y electrocardiográfica de un síndrome coronario típico y resolución completa en semanas a meses, comenzó a difundirse a partir del reporte de Sato et al., iniciando una ola de informes médicos en el mundo de casos con características similares, hasta ser reconocida como una entidad extremadamente inusual, desconocida hasta el momento, y de modo que para el año 2014 ya existían más de 1.000 casos en la literatura[3],[4]. Hoy se considera que el $2 \%$ de los pacientes con síndrome agudo coronario son en realidad síndrome de Tako-Tsubo, de los cuales el $90 \%$ se presentan en mujeres posmenopáusicas desencadenado por un factor de estrés emocional[3] y con una mortalidad estimada entre el $1 \%$ al $5 \%[5]$.

En cuando a la etiopatogenia de la enfermedad, aún no es claro el mecanismo con el que se produce; sin embargo, han podido dilucidarse variantes genéticas en reguladores de proteínas que podrían indicar predisposición del individuo; algunos estudios incluyen alteraciones en el gen de $\mathrm{Bcl}-2$ y polimorfismos de las proteínas $\mathrm{G}$, y los receptores adrenérgicos alfa y betacardiacos[6].

Algunas teorías sobre los mecanismos de producción del aturdimiento miocárdico son: espasmo co- ronario, trastornos de la microcirculación, disfunción miocárdica secundaria a toxicidad por catecolaminas, con hiperactividad aguda del sistema simpático local que sería el desencadenante en entornos críticos[7]. La teoría catecolaminérgica ha sido hasta el día de hoy la más aceptada, partiendo de un factor estresante emocional o físico que desencadena la secreción y liberación de epinefrina y norepinefrina en sangre y, con ello, aumento de la demanda de oxígeno miocárdico y disfunción microvascular; dicha teoría ha sido respaldada por la concentración de catecolaminas en estos pacientes 2 a 3 veces superior a las encontradas en el síndrome coronario. Esta propuesta va de la mano con la teoría de "tráfico de estímulo" en la que los receptores beta 2 estimulados por el aumento de catecolaminas conlleva al cambio conformacional de las proteínas Gs a Gi, lo que induce disminución de la función contráctil; a su vez, estudios de laboratorio han mostrado mayores concentraciones de dichos receptores a nivel del ápex lo que explica la selectividad en la disfunción apical característica de la enfermedad[5],[7]-[9]. Nef et al., demostraron también la participación de la vía fosfatidil inositol 3-quinasaproteína-quinasa (PI3K/AKT) durante la patogénesis, aumentando su función como regulador de isquemia miocárdica, evitando así la apoptosis de los miocitos[10]. Un factor a tener en cuenta es la expresión de receptores de estrógenos alfa y beta en las células cardiacas con función de transcripción de factores cardioprotectores contra el estrés y la toxicidad catecolaminérgica, por lo cual la presentación en mujeres, anterior a la menopausia como en nuestro caso es extremadamente rara[4].

La presentación clínica está dada por disnea y dolor torácico de forma consistente e indistinguible de un infarto agudo de miocardio, sin embargo, otras presentaciones son los signos de falla cardiaca, la hipotensión arterial y el shock cardiogénico, incluso la pérdida de la conciencia previo estrés emocional. Nuestra paciente debutó con hipoxia, hipotensión severa y edema agudo de pulmón, sin embargo, no existe una prevalencia de estos signos. En el caso de un edema agudo de pulmón es una emergencia médica definida como acumulación de líquido en los alveolos y el intersticio pulmonar que puede clasificarse: no cardiogénico (como: edema pulmonar por presión negativa) y cardiogénico asociado en síndrome de Tako-Tsubo posanestésico a sobrecarga de líquidos perioperatorio y estados de predisposición de sobrecarga de volumen[11]-[13].

En el registro electrocardiográfico se muestran cambios inespecíficos por lo cual se han tratado de establecer criterios diagnósticos y de exclusión de la 
enfermedad por varios grupos de investigación de miocardiopatías; sin embargo, los criterios de Mayo establecidos en 2004 y modificados en 2008, siguen siendo los más aceptados, en los que la ausencia de lesión coronaria es un punto clave en la sospecha diagnóstica. Recientemente, fueron ampliados y modificados nuevamente por Lyon et al. en consenso de expertos con la Sociedad Europea de Cardiología en el año 2018[5],[14],[15].

Al día de hoy, se han establecido 5 variaciones de la enfermedad: la forma clásica (80\%), medioventricular $(15 \%)$, reversa o basal (2\%-5\%), tipo focal $(1,5 \%)$ y las formas atípicas (incluida la que afecta el ventrículo derecho)[5]. Nuestro caso es una variante de discinesia medioventricular poco frecuente, descrita inicialmente en el año 2007 con reporte de 5 casos[16], que al comprometer la pared media suele presentar ondas $T$ invertidas en el electrocardiograma, que en este caso no se presenta[17].
Algunos reportes de casos han mostrado relación del Tako-Tsubo y la anestesia, explicada por la descarga catecolaminérgica desencadenada por factores como: manipulación de la vía aérea, el dolor, la extubación, la relajación residual y el recuerdo intraoperatorio[18],[19]. Además, algunos factores que predisponen condiciones cardiacas (asma, cirugía, trauma, hipertiroidismo y hemorragia subaracnoidea) pueden también desencadenar formas secundarias de TakoTsubo[5]. En el caso de nuestra paciente estos factores pudieron influir en la presentación de la entidad, sumado a polimorfismos genéticos familiares preexistentes para la presentación de enfermedad cardiaca temprana.

El diagnóstico estuvo limitado por la presentación poco frecuente de la enfermedad en una mujer joven, además de la predominancia de la falla ventilatoria y la hipotensión arterial severa, con edema pulmonar agudo en un posoperatorio inmediato.

\section{Referencias}

1. Ryan TJ, Fallon JT. Case records of the Massachusetts General Hospital. Weekly clinicopathological exercises. Case 18-1986.

A 44-year-old woman with substernal pain and pulmonary edema after severe emotional stress. N Engl J Med. 1986 May;314(19):1240-7. https://doi.org/10.1056/ NEJM198605083141908 PMID:3702920

2. Sato H, Tateishi H, Dote K, Uchida T, Ishihara M. Tako-tsubolike left ventricular dysfunction due to multivessel coronary spasm. In: Kodama K, Haze K, Hon M, editors. Clinical Aspect of Myocardial Injury: From Ischemia to Heart Failure. Kagakuhyouronsha (Tokyo); 1990. pp. 56-64.

3. Parodi G, Del Pace S, Carrabba N, Salvadori C, Memisha G, Simonetti l, et al. Incidence, clinical findings, and outcome of women with left ventricular apical ballooning syndrome. Am J Cardiol. 2007 Jan;99(2):182-5. https://doi.org/10.1016/j. amjcard.2006.07.080 PMID:17223415

4. Komamura K, Fukui M, Iwasaku T, Hirotani S, Masuyama T. Takotsubo cardiomyopathy: Pathophysiology, diagnosis and treatment. World J Cardiol. 2014 Jul;6(7):602-9. https:// doi.org/10.4330/wjc.v6.i7.602 PMID:25068020

5. Dias A, Núñez Gil IJ, Santoro F, Madias JE, Pelliccia F, Brunetti ND, et al. Takotsubo syndrome: state-of-the-art review by an expert panel - Part 1. Cardiovasc Revasc Med. 2019 Jan;20(1):709. https://doi.org/10.1016/j. carrev.2018.11.015 PMID:30528096

6. Y-Hassan S, De Palma R. Contemporary review on the pathogenesis of takotsubo syndrome: The heart shedding tears: Norepinephrine churn and foam at the cardiac sympathetic nerve terminals. Int J Cardiol. 2017 Feb;228:528-36. https://doi. org/10.1016/j.ijcard.2016.11.086 PMID:27875730
7. Bybee KA, Kara T, Prasad A, Lerman A, Barsness GW, Wright RS, et al. Systematic review: transient left ventricular apical ballooning: a syndrome that mimics ST-segment elevation myocardial infarction. Ann Intern Med. 2004 Dec;141(11):858-65. https://doi.org/10.7326/00034819-141-11-200412070-00010 PMID:15583228

8. Y-Hassan S. Acute cardiac sympathetic disruption in the pathogenesis of the takotsubo syndrome: a systematic review of the literature to date. Cardiovasc Revasc Med. 2014 Jan;15(1):3542. https://doi.org/10.1016/j. carrev.2013.09.008 PMID:24140050

9. Gianni M, Dentali F, Grandi AM, Sumner G, Hiralal R, Lonn E. Apical ballooning syndrome or takotsubo cardiomyopathy: a systematic review. Eur Heart J. 2006 Jul;27(13):1523-9. https:// doi.org/10.1093/eurheartj/ ehl032 PMID:16720686

10. Nef HM, Möllmann $H$, Hilpert P, Troidl C, Voss S, Rolf A, et al. 
Activated cell survival cascade protects cardiomyocytes from cell death in Tako-Tsubo cardiomyopathy. Eur J Heart Fail. 2009 Aug;11(8):758-64. https:// doi.org/10.1093/eurjhf/hfp076 PMID:19633102

11. Edinson $G$, Gustavo R, Cubides CA, Múnera AG, Dagnóvar A. Miocardiopatía por estrés (miocardiopatía tipo takotsubo): presentación de un caso clínico y revisión de la literatura. Rev. Col. Cardiol. [Internet]. 2006 Aug [cited 2020 Aug 06]; 13 (1) : 31-38. Available from: http://www.scielo.org. co/scielo. php?script=sci_ arttext\&pid=S012056332006000400006\&lng=en

12. Varutti $R$, Setti T, Ezri T, Nicolosi G, Rellini G, Cassin M, et al. Postoperative Takotsubo cardiomyopathy triggered by intraoperative fluid overload and acute hypertensive crisis. Rom J Anaesth Intensive Care. 2015 Apr;22(1):47-50. PMID:28913455

13. Sureka B, Bansal K, Arora A.
Pulmonary edema - cardiogenic or noncardiogenic? J Family Med Prim Care. 2015 Apr-Jun;4(2):290. https://doi. org/10.4103/2249-4863.154684 PMID:25949989

14. Lyon $A R$, Bossone $E$, Schneider $B$, Sechtem U, Citro R, Underwood SR, et al. Current state of knowledge on Takotsubo syndrome: a Position Statement from the Taskforce on Takotsubo Syndrome of the Heart Failure Association of the European Society of Cardiology. Eur J Heart Fail. 2016 Jan;18(1):8-27. https://doi.org/10.1002/ejhf.424 PMID:26548803

15. Pilgrim TM, Wyss TR. Takotsubo cardiomyopathy or transient left ventricular apical ballooning syndrome: A systematic review. Int J Cardiol. 2008 Mar;124(3):28392. https://doi.org/10.1016/j. ijcard.2007.07.002 PMID:17651841

16. Núñez Gil IJ, García-Rubira JC, Luaces Méndez M, FernándezOrtiz A, Vivas Balcones D, González Ferrer JJ, et al.
[Transient left midventricular dyskinesia: clinical features of a new variant]. Rev Esp Cardiol. 2008 Mar;61(3):322-6. PMID:18361908

17. Kurowski $V$, Kaiser $A$, von Hof K, Killermann DP, Mayer B, Hartmann F, et al. Apical and midventricular transient left ventricular dysfunction syndrome (tako-tsubo cardiomyopathy): frequency, mechanisms, and prognosis. Chest. 2007 Sep;132(3):809-16. https://doi. org/10.1378/chest.07-0608 PMID:17573507

18. Meng L, Wells $C$. Takotsubo cardiomyopathy during emergence from general anaesthesia. Anaesth Intensive Care. 2009 Sep;37(5):836-9. https://doi.g/1 $0.1177 / 0310057$ X0903700518 PMID:19775052

19. Lopera L, Restrepo C, Lopera O, Mendoza J. Cardiomiopatía Takotsubo en la unidad de cuidado postanestésico. Revista Colombiana de Anestesiología. 2012;40(3):240-4. https://doi. org/10.1016/j.rca.2012.05.003. 